OPEN ACCESS

Edited by: Antonio Calles,

Gregorio Marañón Hospital, Spain

Reviewed by:

Alessandra Curioni Fontecedro, University of Zurich, Switzerland Elena Levantini,

Beth Israel Deaconess Medical Center, Harvard Medical School, United States

*Correspondence: Joseph R. Testa joseph.testa@fccc.edu Anton Berns a.berns@nki.n

Specialty section:

This article was submitted to

Thoracic Oncology,

a section of the journal

Frontiers in Oncology

Received: 17 October 2019 Accepted: 20 January 2020

Published: 11 February 2020

Citation:

Testa JR and Berns A (2020) Preclinical Models of Malignant Mesothelioma. Front. Oncol. 10:101.

doi: 10.3389/fonc.2020.00101

\section{Preclinical Models of Malignant Mesothelioma}

\author{
Joseph R. Testa ${ }^{1 *}$ and Anton Berns ${ }^{2 *}$ \\ ${ }^{1}$ Cancer Biology Program, Fox Chase Cancer Center, Philadelphia, PA, United States, ${ }^{2}$ Division of Molecular Genetics, The \\ Netherlands Cancer Institute, Amsterdam, Netherlands
}

Rodent models of malignant mesothelioma help facilitate the understanding of the biology of this highly lethal cancer and to develop and test new interventions. Introducing the same genetic lesions as found in human mesothelioma in mice results in tumors that show close resemblance with the human disease counterpart. This includes the extensive inflammatory responses that characterize human malignant mesothelioma. The relatively fast development of mesothelioma in mice when the appropriate combination of lesions is introduced, with or without exposure to asbestos, make the autochthonous models particularly useful for testing new treatment strategies in an immunocompetent setting, whereas Patient-Derived Xenograft models are particularly useful to assess effects of inter- and intra-tumor heterogeneity and human-specific features of mesothelioma. It is to be expected that new insights obtained by studying these experimental systems will lead to new more effective treatments for this devastating disease.

Keywords: malignant mesothelioma, preclinical rodent models, in vivo asbestos carcinogenesis, genetic driver lesions, mesothelioma inflammatory phenotype, conditional tumor suppressor gene knockout/oncogene mouse models, patient-derived xenograft models of mesothelioma

\section{INTRODUCTION}

Malignant mesothelioma (MM) is a treatment-resistant malignancy causally linked to asbestos exposure. Despite recent advances in therapeutic modalities, MM patients usually die within 1 year following diagnosis. MM is particularly lethal in patients with pleural disease, particularly those whose tumors have sarcomatoid features (1). Consequently, in vivo models of MM are needed to investigate MM disease pathogenesis and to provide accurate preclinical models for identifying new therapies that might move forward in clinical trials.

We here summarize where we stand with regard to existing models of MM and how they might be further improved. All the desirable features will be unlikely found in a single model, but the disease evolving in the model should mimic at least several of the salient features of human MM, such as its pathology, its gene expression patterns, the genetic driver lesions, and the inflammatory phenotype that is characteristic for MM. In view of the inflammatory phenotype of MM and the prominent role the immune system fulfills in either promoting or impairing tumor development, models exhibiting this specific feature should also be part of the armamentarium. Preferentially, the model should also exhibit a reproducible and short latency period as to permit intervention studies. The models-mostly encompassing small rodents-range from graft models in which human MM cell lines or patient-derived tumor fragments are implanted to complex conditional tumor suppressor gene knockout/oncogene mouse models. 


\section{SOMATIC GENETIC AND SIGNALING ALTERATIONS IN HUMAN MESOTHELIOMA}

There is abundant evidence that inactivating somatic mutations and deletions of the tumor suppressor genes (TSGs) BAP1, CDKN2A, and NF2 represent the most frequent genetic lesions in human malignant pleural mesothelioma (MPM) (2-13). Moreover, losses of these three TSGs are frequently seen in various combinations in a given MPM $(7,14)$. The notion that loss of these particular TSGs is so predominant implies that MPM development critically depends on the cellular signaling pathways that are guarded by these genes.

CDKN2A encodes p16INK4A and p14ARF, two tumor suppressors that, respectively, regulate the $\mathrm{Rb}$ and $\mathrm{p} 53$ cell cycle pathways. p14ARF is a component of the p53 pathway, and TP53 alterations have also been observed in some MPMs $(6,15)$. In fact, a recent report that compared next-generation sequencing of two series of MPMs-one from The Cancer Genome Atlas (TCGA) (13) and the second from a Harvard series (12) - revealed only four "significantly mutated genes at a false discovery rate of $<0.05$ " common to the two studies: BAP1, NF2, TP53, and SETD2, each of which showed prominent levels of inactivating nonsense, frameshift, and splice-site mutations, consistent with their putative roles as driver loss-of-function lesions in this malignancy (13). In the TCGA data set, focal deletions were found to affect several TSGs, especially CDKN2A, with deep, apparently homozygous deletions occurring in 36/73 (49\%) tumors and single-copy losses in 5 others (7\%) (13). In the Harvard series, Bueno et al. found copy number losses of CDKN2A in 48/95 (51\%) MPMs (12). In a deletion mapping analysis, homozygous $C D K N 2 A$ deletions were identified in 36 of 40 (90\%) human MPM cell lines tested, while homozygous deletions of the adjacent locus $C D K N 2 B$ occurred in mosti.e., 32/36-of these same cell lines (6). Experiments in mice have shown that the $C d k n 2 b$ also exhibits a tumor suppressor role in MPM, as its deletion concomitant with Cdkn2a further accelerates MPM development (our unpublished results) offering a rationale for the predominant deletion of all three tumor suppressors in this locus in MPM.

Unlike these specific TSGs, mutations of protooncogenes are seldom identified in MPM. Moreover, in the TCGA cohort, no activating mutations were observed in genes encoding components of the MAPK or PI3K/AKT pathways (13). However, both PI3K/AKT/mTOR and RAS/MAPK pathways were upregulated in this series, and they were each associated with a poor-prognosis. Moreover, despite a rarity of mutations of PTEN in MPM, earlier immunohistochemical (IHC) studies revealed diminished PTEN protein expression in 16 to $62 \%$ of MMs in several studies (16-18). Additionally, various receptor tyrosine kinases (RTKs) were shown to be frequently overexpressed and/or activated in MPM, resulting in activation of proliferation and pro-survival signals through the PI3K/AKT/mTOR signaling pathway $(7,19-21)$. Thus, it is not surprising that phospho-AKT immunostaining is observed in a high percentage (65-84\%) of human MPMs $(6,16,22,23)$.
In view of the prominence of TSG inactivation and the relatively rare oncogenic gain-of-function mutations in MM, high-throughput chemical inhibitor screens and gene expression analyses have been performed in MM cell lines to identify unique vulnerabilities. Chemical screens pointed to increased sensitivity to FGFR inhibitors in a subset of the MPM cell lines. This corresponded with higher FGFR3 expression specifically in cell lines not expressing BAP1 (24). BAP1-deficient MM also showed augmented sensitivity to TRAIL (25). Furthermore, loss of BAP1 function was found associated with increased expression of EZH2, with concomitant widespread epigenetic gene silencing sensitizing the cells to EZH2 inhibitors (26), whereas the impaired argininosuccinate synthase 1 (ASS1) expression likely as a result of enhanced EZH2 levels sensitized cells to arginine deprivation $(27,28)$. In addition, BAP1-depleted cells showed increased sensitivity to PARP inhibition (29). Another vulnerability relates to the co-deletion of CDKN2A and the nearby methylthioadenosine phosphorylase (MTAP) gene (30), the latter rendering cells dependent on protein arginine methyltransferase (PRMT5) (31, 32). NF2 depletion leads to dysregulation of the Hippo pathway by activating the transcriptional co-activator YAP1 and its association with the TEAD family of transcription factors, resulting in up-regulation of genes that promote cell proliferation and inhibit cell death. Inhibitors that disrupt the YAP/TAZ-TEAD complex are not yet available but could serve as promising drugs in view of the strong dependence of MM on activation of the Hippo pathway (33). MM also shows overexpression of RTKs such as MET and downstream PI3K, making inhibitors targeting components of this pathway other promising therapies for this disease (21). Therefore, there are a number of potential vulnerabilities that are worth exploring both as single agents and as combinations in the various preclinical models of MM.

\section{RODENTS AS MODELS OF ASBESTOS CARCINOGENICITY AND MESOTHELIOMA PATHOGENESIS}

Numerous investigators have induced MM in rats and mice via injection or inhalation of asbestos fibers (34) or in hamsters through exposure to SV40 (35). Notably, several studies have shown that the MMs induced in rats via asbestos inhalation do not exhibit cytogenetic or gene expression patterns similar to those seen in their human tumor counterparts nor do they show inactivation of genes implicated as drivers in human MM (36-39). Studies in the laboratory rat, beginning in the 1960's, documented that various forms of asbestos and other mineral fibers inoculated intrapleurally/intrathoracically (IT) developed MPM (40). Erionite, the zeolite mineral fiber that is linked to the MM epidemic in near Cappadocia, Turkey $(41,42)$ was shown to be more carcinogenic than asbestos in IT injection or inhalation studies (43).

While the rat was favored over the mouse as a model for mineral fiber studies due to its larger pleural space for inoculation and "... its more suitable nasal passage architecture 
for inhalation studies, some of the early investigations did use mice for IT inoculation of amphiboles and serpentine mineral fibers," however, fibrosis and granulomas were mainly observed (44), with occasional papillary carcinomas seen in inhalation experiments (45). Subsequent carcinogenicity studies using intraperitoneally (i.p.)-inoculated asbestos or zeolite fibers resulted in MMs in more than $20 \%$ of wild type mice (46). Over the last two decades, various laboratories have reported variable MM incidences and survival rates in wild type mice that have been injected i.p. with asbestos fibers $(6,38,47-56)$, due at least in part to the use of differing types, dimensions and amounts of fibers used, whether the injections were given chronically or as a bolus injection, the length of time the animals were followed, and variations in the genetic background of the mice.

Genetically engineered mouse (GEM) models, typically harboring heterozygous whole-body germline mutations, have been used to assess whether loss of TSGs implicated in human MPM accelerate tumor formation. Different groups have performed such experiments with GEM models carrying mutations of MM-related genes. An early investigation used Tp53- deficient mice $(38,47)$, with mice injected i.p. with crocidolite weekly for 22 weeks. Tp53 $3^{+/-}$mice developed a high incidence $(76 \%)$ of MMs (median latency, 44 weeks) vs. a32\% of wild type mice (median latency, 67 weeks). Only 1/8 (12.5\%) Tp53-/- mice had a MM, with others succumbing quickly due to thymic lymphomas or hemangiosarcomas, previously reported to arise spontaneously in $T p 53^{-/-}$mice (57).

Two research groups tested whether heterozygous $N f 2$ mice have increased susceptibility to the carcinogenic effects of asbestos $(6,48)$. Both groups independently demonstrated that $N f 2^{+/-}$mice injected i.p. with asbestos develop a high incidence and rapid onset of MMs compared wild type littermates. Notably, the normal $N f 2$ allele was deleted in most MMs from the $N f 2^{+/-}$ mice, consistent with biallelic inactivation, which similarly occurs in many human MPMs (6). Moreover, most MM cell lines from the Nf2-deficient mice showed homologous deletions of $C d k n 2 a / C d k n 2 b$ and activation of Akt, recapitulating events that often occur in human MPM. Collectively, these findings are consistent with Nf2 being a TSG that, when inactivated, acts as a primary driver in the formation of $\mathrm{MM}$.

As noted previously, CDKN2A encodes p16INK4A and p14ARF (19Arf in the mouse). To test the relative contributions of these genes to MM formation, one study used mice with heterozygous deletions of Cdkn2a exon $1 \alpha$ (resulting in loss of p16Ink4a) or exon $1 \beta$ (p19Arf), or with a deletion of exon 2 (deleting both p16Ink4a and p19Arf) (51). Both p16Ink4a $a^{+/-}$ mice and $p 19 A r f^{+/-}$mice injected i.p. with asbestos exhibited higher incidence and more rapid onset of MM than wild type control mice. Mice heterozygous for $C d k n 2 a$ exon 2 showed a more accelerated rate of asbestos-induced MMs vs. mice deficient for either p16Ink4a or p19Arf separately. Together, these data indicate that each of the Cdkn2a gene products suppresses asbestos-induced $\mathrm{MM}$, and that the combined inactivation of both gene products results in further cooperation to accelerate asbestos-induced MM development and progression.

Early Sanger sequencing studies had revealed point mutations in BAP1 in $20-25 \%$ of sporadic human MMs
(7, 8), but subsequent studies of sporadic MMs using various combinations of assays, such as quantitative real-time PCR, targeted comparative genomic hybridization, next generation sequencing, and/or multiplex ligation-dependent probe amplification platforms demonstrated $B A P 1$ alterations in up to $60-65 \%$ of MMs (9-11). Most of the alterations not detected by Sanger sequencing were large deletions.

In addition to somatic changes, it is now well-established that $B A P 1$ mutation carriers are predisposed to $\mathrm{MM}$ and a variety of other tumors $(8,58)$. The use of Bap1 knockout models has shown that heterozygosity in the germline predisposes to asbestos-induced MM $(53,59)$, and similar results were obtained with two knock-in models (54) that harbored different germline mutations that were identical to the ones found in two BAP1 tumor predisposition syndrome (BAP1-TPDS) families that exhibited a very high incidence of MM (8). MM cells from $B a p 1^{+/-}$mice showed biallelic inactivation of Bap1 (53). Collectively, these data indicate that human BAP1 mutation carriers have are more prone to the carcinogenic effects of asbestos, even when exposed to small amounts of these fibers (59), when compared to the general population.

Other work has recently demonstrated cooperation between Nf2 and Cdkn2a in MM development in asbestos-exposed $N 2^{+/-}$;Cdkn $2 a^{+/-}$mice, which exhibited significantly hastened tumor onset and disease progression vs. similarly exposed $N f 2^{+/-}$and wild-type cohorts (56). These studies also showed that tumors from $N f 2^{+/-} ; C d k n 2 a^{+/-}$mice had enhanced metastatic potential and an increased cancer stem cell population, in connection with p53/miR-34a-dependent activation of c-Met.

Since chronic inflammation may contribute to the formation of many types of malignancy, including MM, some investigators have employed mouse models for studies of asbestos-mediated inflammation. In one such study, $\mathrm{Nf2} 2^{+/-} ; C d k n 2 a^{+/-}$mice were used to test if inflammation-related IL- $1 \beta$ release promotes MM formation (55). Exposure of $N f 2^{+/-}$; Cdkn2a $a^{+/-}$mice to asbestos in the presence of an IL-1 receptor (IL-1R) antagonist known as anakinra resulted in a significant delay MM development compared to that of asbestos-exposed mice given a vehicle control, i.e., 33 vs. $\sim 22.5$ weeks, respectively (55). Overall, this work suggested that inflammation-related IL-1 $\beta /$ IL-1R signaling is linked to the formation of asbestos-induced MM. Moreover, the data demonstrate the usefulness of this model for geneenvironment and/or "chemoprevention" studies.

Another mouse model, MexTAg, has been used to demonstrate co-carcinogenicity between asbestos and SV40. The investigators used the mesothelin gene promoter to express SV40 large $\mathrm{T}$ antigen specifically in the mesothelial lining $(49,60)$. Several MexTAg mouse lines were created with varying copies (1-100) of the oncogenic transgene. The animals generally do not develop spontaneous MM. However, after i.p. injection of asbestos, $100 \%$ of the MexTAg mice developed MM, with disease onset occurring after 20-40 weeks vs. after 50-100 weeks in the $\sim 25 \%$ of wild type mice that developed MM. The investigators concluded that MexTAg mice are well-suited not only basic research, but also for testing the potential of dietary or pharmacological chemoprevention studies of MM (49). To illustrate the utility of MexTAg mice for preclinical studies, 
Robinson et al. tested the effects of gemcitabine, a cytotoxic drug that has been shown to have some efficacy in the human disease (60). MexTAg mice treated with vehicle had a median survival of 33 vs. 48 weeks in the gemcitabine-treated cohort. In another investigation with MexTAg mice, treatment with celecoxib, a COX-2 inhibitor, did not diminish the rate of asbestos-induced MM, despite the fact that COX-2 is frequently overexpressed in human MM and correlates with poor prognosis (60). While the MexTAg model has several advantages (100\% MM penetrance, short median survival), it does not have any of the genetic hallmarks attributed to the human disease, and a causative association between SV40 and human MM is now disproven $(61,62)$. However, in one study, gene expression profiling of MMs from MexTAg mice “...had a concordant set of deregulated genes compared to normal mesothelial cells that overlapped with the deregulated genes between human MMs and mesothelial cells" (63).

\section{CONDITIONAL MOUSE MODELS OF MESOTHELIOMA AS PRECLINICAL TOOLS}

Since specific genetic driver lesions had been repeatedly found to be associated with human MM by the year 2008, particularly alterations of the CDKN2A, NF2, and TP53, Jongsma et al. decided to establish whether various genetic alterations affecting the same signaling pathways that are dysregulated in the human disease counterpart might similarly induce MM in rodents in the absence of carcinogenic exposure to asbestos (64). Thus, they generated a variety of mutant mice carrying deletions in the $\mathrm{Nf} 2 /$ merlin, $\mathrm{p} 53$, and/or Ink4a pathways, hypothesizing that mice with one or more of these combinations might represent an appropriate model of human MM. To avoid possible issues such as embryonic lethality due to germline homozygous deletion of one or more targeted genes, mice with conditional knockout (CKO) of various TSGs were used in combination with the Cre-LoxP system (65). Locotemporal inactivation of the TSG(s) was carried out by injecting adenoviruses expressing the Cre recombinase (65). Upon injecting adeno-Cre into the pleural space of Rosa26 LacZ reporter mice, the investigators demonstrated expression of $\beta$-galactosidase specifically in the mesothelium (64). Moreover, MPMs arose in both Nf2;Tp53 and Nf2;p16Ink4a/p19Arf CKO mice at a high frequency and short latency (20 and 30 weeks, respectively) following IT inoculation of adeno-Cre, and the tumors closely mimicked the phenotype of human MPM. Thus, these mice hold promise as a rapid, non-carcinogenic model system for preclinical selection of new combination therapies and for testing novel targeted agents.

BAP1-TPDS patients with MM have a significantly better long-term survival compared to sporadic MM patients, i.e., those without a heritable variant $(11,66)$. However, it remained unclear whether somatic mutations/deletions of BAP1 have a similarly favorable prognosis in sporadic MM, or if somatic $B A P 1$ alterations are a poor prognostic marker, as is the case for uveal melanoma and clear cell renal cell carcinoma (67, 68). Furthermore, although most human MMs exhibit somatic alterations of $B A P 1, N F 2$, and/or $C D K N 2 A$-with 25/74 cases of MPM in the TCGA series having alterations of all three TSGs in combination (13) - it was not known if loss of BAP1 could cooperate with the inactivation of NF2 and/or CDKN2A to initiate a more aggressive form of MM. To address this possibility experimentally, Kukuyan et al. used CKO models, including a $B a p 1^{f / f}$ mouse they generated (69). Various combinations of deletions of Bap1, Cdkn2a, and $N f 2$ were introduced in the pleural cavity of the mice, focusing on the contribution of Bap1 loss. While homozygous CKO of any one of these TSGs alone gave rise to few or no MMs-similar to the results of Jongsma et al. (64) - deletion of Bap1 cooperated with deletion of either Nf2 or $C d k n 2 a$ to promote MM formation in about $20 \%$ of doubleCKO mice. In contrast, a much higher incidence $(22 / 26,85 \%)$ of MMs was observed in $B a p 1^{f / f} ; N f 2^{f / f}$;Cdkn2a $a^{f / f}$ mice injected IT with adeno-Cre (triple-CKO mice). Onset of MM was rapid in the triple-CKO mice (median survival, 12 weeks), and tumors from these mice were consistently high-grade and invasive. With regard to histological subtype, notably no epithelioid MMs were observed with any of the mouse genotypes. Sarcomatoid MMs predominated, with the only exception being the Bap1;Nf2 double-CKO cohort, in which 6 of 7 MMs showed mixed (biphasic) histology. The MMs observed in triple-CKO mice showed enrichment for genes that are transcriptionally controlled by the polycomb repressive complex 2 (PRC2) (69). The findings suggested that loss of Bap1 contributes to MM progression, at least partially, via loss of PRC2-mediated repression of oncogenic target genes that were identified, suggesting a novel avenue for therapeutic intervention (69).

To explore the role of individual components of the $C d k n 2 a$ locus by comparing models in which $C d k n 2 a$ (including p19Arf) were disrupted with or without concomitant loss of $C d k n 2 b$ Badhai et al. showed that the additional disruption of $C d k n 2 b$ further added to the aggressiveness of the resulting MMs, providing also an explanation for the predominance of deletion of the complete $C D K N 2 A-C D K N 2 B$ locus in human MM over point mutations in $C D K N 2 A$ (Badhai et al., submitted).

Because CDKN2A deletions encompassing the sequence encoding p14ARF, a component of the p53 pathway, have been documented in $90 \%$ of human MM cell lines (6) and TP53 is altered in about $15 \%$ of primary MMs, and because the $\mathrm{PI} 3 \mathrm{~K} / \mathrm{PTEN} / \mathrm{AKT}$ pathway is activated in most human MPMs, Sementino et al. decided to determine if alterations affecting the same pathways would also induce MM in mice (70). This was thought worthwhile, given that p53 helps mediate the DNA damage response and that AKT regulates neoplastic cell survival and therapeutic resistance. The investigators demonstrated that while neither adeno-Cre-mediated homozygous deletion of Tp53 or Pten alone in the mesothelium was sufficient to induce MM formation, compound deletion of these two TSGs resulted in rapid, aggressive peritoneal and pleural MMs (median latency: 9 and 19 weeks, respectively). A longer term follow-up study of the Tp53f/f cohort revealed MMs in 0/12 mice injected with adenoCre i.p. and 0/10 mice injected IT; among the Pten $f / f$ cohort, MMs were observed in 0/12 mice injected i.p. and $1 / 10$ injected IT (Sementino et al., unpublished data). In the Pten $f / f ; T p 53^{f / f}$ cohort, 23/25 (92\%) mice injected i.p. developed MM, whereas 
19/34 (56\%) mice injected IT showed MM, with 14 histiocytic sarcomas also seen in this group.

Given the high penetrance and rapid development of MMs in Pten ${ }^{f / f} ; T p 53^{f / f}$ mice inoculated i.p., and the frequent involvement of p14ARF/p53 and PI3K/PTEN/AKT pathways in human MM, this GEM model holds promise for preclinical work. However, this model does have certain limitations, such as for testing agents designed to reactivate the normal cellular functions of Pten and Tp53. For instance, given that this model has homozygous loss of $T p 53$, this precludes studies of a drug such as RITA, which reactivates p53's pro-apoptotic function in tumor cells that preserve expression of mutant or wild-type p53 (71). To elude this issue using an agent such as RITA, this mouse model might be modified such that only a single $T p 53$ allele were deleted, i.e., by using $P t e n^{f / f} ; T p 53^{+/ f}$ mice. A second shortcoming with regard to the translational relevance of the Pten ${ }^{f / f} ; T p 53^{f / f}$ model is that somatic mutations of other TSGs considered to be hallmarks in human MM progression usually do not occur in tumors from these animals. However, the fact that the MMs in this model repeatedly show sarcomatoid or biphasic histology with very short latency, especially in mice injected i.p., provides advantages for certain preclinical applications.

\section{GRAFT MODELS OF MESOTHELIOMA}

Many human MM cell lines have been established over the years and used in numerous in vitro studies. They are also exploited for in vivo experiments, usually for testing their tumorigenicity and the efficacy of small molecule inhibitors as a prelude for evaluating these compounds in clinical trials. Due to often longterm in vitro propagation, these cell lines have invariably acquired (epi)genetic alterations that facilitate their propagation in cell culture, resulting in new vulnerabilities and resistance features. This is one of the reasons why treatments that are effective in these graft models often do not well-translate to human. Furthermore, the requirement to use immunodeficient mice as a host for these graft experiments complicates assessment of immunomodulating effects. Patient-derived xenograft (PDX) models, in which tumor fragments are grafted directly into immunodeficient recipient hosts, more closely resemble the human condition and usually retain their human stromal components for a number of passages. The capacity to establish PDX lines also correlates with the aggressiveness of the tumor in man (72). Studies in PDX models permit addressing specific questions that are difficult to assess in solely mouse based models such as inter- and intra-tumor heterogeneity as well as features imposed by the distinct genetic backgrounds (73). As potential drawbacks, we note that propagation has to be performed in immunodeficient backgrounds and retrofitting these models with a functional human immune system from the patient from which the tumor was obtained (humanized models) is still in an early stage of development (74) and also practically very demanding.

Experienced investigators seeking a mouse model that may faithfully reflect human MPM pathobiology may also find an orthotopic, intrapleural model such as the one described by Servais et al. (75) useful for preclinical therapeutic studies. This tumor model recapitulates human pleural anatomy/microenvironment and can be used in combination with quantitative, non-invasive imaging for bioluminescent monitoring of tumor burden. The parietal pleural surface contains lymphatics that offer escape of MM cells into the systemic circulation, and this immunocompetent orthotopic model of pleural cancer permit studies of inflammation on tumor progression as well (75). However, as noted by the authors, for studies of therapies targeting human antigens, immunodeficient models are required in order to perform studies on xenografted human cancer cell lines.

\section{ARE WE MISSING ANYTHING?}

First of all, it is worth emphasizing that the choice of the model depends on the question asked. Furthermore, a mouse is not a "small human" and we need to accept that we cannot simply extrapolate findings from such a model to the human condition. However, models can teach us important biological principles and can provide us with therapeutic concepts worth testing in clinical settings notwithstanding the evolutionary distance between man and mouse. Where possible, we should try to align the model on the basis of molecular aberrations found in humans, e.g., by introducing similar driver lesions in the right target cell and using comparable external carcinogens if applicable, e.g., asbestos. Evidently, PDX models might be very valuable to assess intrinsic tumor heterogeneity and to evaluate their response to drug combinations. For immunotherapy studies, it will be important to use a model with a functional immune system. To permit effective immunotherapy studies in MM mouse models, it will be important to establish these in a defined genetic background (e.g., BL6, the "work horse" of immunologists) in order to permit isogenic graft studies. Fortunately, current Crispr/Cas9 engineering has made the generation of complex conditional MM models relatively easy. This should facilitate the testing of new promising intervention strategies for this highly lethal cancer.

\section{AUTHOR CONTRIBUTIONS}

$\mathrm{JT}$ and $\mathrm{AB}$ wrote the paper and are accountable for the content of the work.

\section{FUNDING}

JT was supported in part by NCI grants CA175691 and CA06927, an appropriation from the Commonwealth of Pennsylvania, and the Local \#14 Mesothelioma Fund of the International Association of Heat and Frost Insulators and Allied Workers. AB was supported by European Research Council Grant 319661 COMBATCANCER and The Queen Wilhelmina Prize from the Dutch Cancer Society. 


\section{REFERENCES}

1. Amin W, Linkov F, Landsittel DP, Silverstein JC, Bashara W, Gaudioso C, et al. Factors influencing malignant mesothelioma survival: a retrospective review of the National Mesothelioma Virtual Bank cohort [version 3]. F1000Res. (2018) 7:1184. doi: 10.12688/f1000research.15512.2

2. Cheng JQ, Jhanwar SC, Klein WM, Bell DW, Lee W-C, Altomare DA, et al. p16 alterations and deletion mapping of 9p21-p22 in malignant mesothelioma. Cancer Res. (1994) 54:5547-5551.

3. Xio S, Li D, Vijg J, Sugarbaker DJ, Corson JM, Fletcher JA. Codeletion of p15 and p16 in primary malignant mesothelioma. Oncogene. (1995) 11:511-5.

4. Bianchi AB, Mitsunaga S-I, Cheng JQ, Klein WM, Jhanwar SC, Seizinger B, et al. High frequency of inactivating mutations in the neurofibromatosis type 2 gene (NF2) in primary malignant mesotheliomas. Proc Natl Acad Sci USA. (1995) 92:10854-8. doi: 10.1073/pnas.92.24.10854

5. Sekido Y, Pass HI, Bader S, Mew DJY, Christman MF, Gazdar AF, et al. Neurofibromatosis type 2 (NF2) gene is somatically mutated in mesothelioma but not in lung cancer. Cancer Res. (1995) 55:1227-31.

6. Altomare DA, Vaslet CA, Skele KL, De Rienzo A, Devarajan K, Jhanwar SC, et al. A mouse model recapitulating molecular features of human mesothelioma. Cancer Res. (2005) 65:8090-5. doi: 10.1158/0008-5472.CAN-05-2312

7. Bott M, Brevet M, Taylor BS, Shimizu S, Ito T, Wang L, et al. The nuclear deubiquitinase BAP1 is commonly inactivated by somatic mutations and 3p21.1 losses in malignant pleural mesothelioma. Nat Genet. (2011) 43:66872. doi: $10.1038 /$ ng. 855

8. Testa JR, Cheung M, Pei J, Below JE, Tan Y, Sementino E, et al. Germline BAP1 mutations predispose to malignant mesothelioma. Nat Genet. (2011) 43:1022-5. doi: 10.1038/ng.912

9. Yoshikawa Y, Sato A, Tsujimura T, Emi M, Morinaga T, Fukuoka K, et al. Frequent inactivation of the BAP1 gene in epithelioid-type malignant mesothelioma. Cancer Sci. (2012) 103:868-74. doi: 10.1111/j.1349-7006.2012.02223.x

10. Lo Iacono M, Monica V, Righi L, Grosso F, Libener R, Vatrano S, et al. Targeted next-generation sequencing of cancer genes in advanced stage malignant pleural mesothelioma: a retrospective study. J Thorac Oncol. (2015) 10:4929. doi: $10.1097 /$ JTO.0000000000000436

11. Nasu M, Emi M, Pastorino S, Tanji M, Powers A, Luk H, et al. High incidence of somatic BAP1 alterations in sporadic malignant mesothelioma. $J$ Thorac Oncol. (2015) 10:565-76. doi: 10.1097/JTO.0000000000000471

12. Bueno R, Stawiski EW, Goldstein LD, Durinck S, De Rienzo A, Modrusan $Z$, et al. Comprehensive genomic analysis of malignant pleural mesothelioma identifies recurrent mutations, gene fusions and splicing alterations. Nat Genet. (2016) 48:407-16. doi: 10.1038/ng.3520

13. Hmeljak J, Sanchez-Vega F, Hoadley KA, Shih J, Stewart C, Heiman D, et al. Integrative molecular characterization of malignant pleural mesothelioma. Cancer Discov. (2018) 8:1548-65. doi: 10.1158/2159-8290.CD-18-0804

14. Cheung M, Testa JR. BAP1, a tumor suppressor gene driving malignant mesothelioma. Transl Lung Cancer Res. (2017) 6:270-8. doi: 10.21037/tlcr.2017.05.03

15. Cote RJ, Jhanwar SC, Novick S, and Pellicer A. Genetic alterations of the p53 gene are a feature of malignant mesothelioma. Cancer Res. (1991) 51:5410-6.

16. Garland LL, Rankin C, Gandara DR, Rivkin SE, Scott KM, Nagle RB, et al. Phase II study of erlotinib in patients with malignant pleural mesothelioma: a Southwest Oncology Group Study. J Clin Oncol. (2007) 25:2406-13. doi: 10.1200/JCO.2006.09.7634

17. Opitz I, Soltermann A, Abaecherli M, Hinterberger M, Probst-Hensch $\mathrm{N}$, Stahel R, et al. PTEN expression is a strong predictor of survival in mesothelioma patients. Eur J Cardiothorac Surg. (2008) 33:5026. doi: 10.1016/j.ejcts.2007.09.045

18. Agarwal V, Campbell A, Beaumont KL, Cawkwell L, Lind MJ. PTEN protein expression in malignant pleural mesothelioma. Tumour Biol. (2013) 34:84751. doi: 10.1007/s13277-012-0615-9

19. Perrone F, Jocolle G, Pennati M, Deraco M, Baratti D, Brich S, et al. Receptor tyrosine kinase and downstream signalling analysis in diffuse malignant peritoneal mesothelioma. Eur J Cancer. (2010) 46:283748. doi: 10.1016/j.ejca.2010.06.130

20. Menges CW, Chen Y, Mossman BT, Chernoff J, Yeung AT, Testa JR. A phosphotyrosine proteomic screen identifies multiple tyrosine kinase signaling pathways aberrantly activated in malignant mesothelioma. Genes Cancer. (2010) 1:493-505. doi: 10.1177/1947601910375273
21. Kanteti R, Dhanasingh I, Kawada I, Lennon FE, Arif Q, Bueno R, et al. MET and PI3K/mTOR as a potential combinatorial therapeutic target in malignant pleural mesothelioma. PLoS ONE. (2014) 9:e105919. doi: 10.1371/journal.pone.0105919

22. Cedres S, Ponce-Aix S, Pardo-Aranda N, Navarro-Mendivil A, Martinez-Marti A, Zugazagoitia J, et al. Analysis of expression of PTEN/PI3K pathway and programmed cell death ligand 1 (PD-L1) in malignant pleural mesothelioma (MPM). Lung Cancer. (2016) 96:1-6. doi: 10.1016/j.lungcan.2016.03.001

23. Altomare DA, Testa JR. Perturbations of the AKT signaling pathway in human cancer. Oncogene. (2005) 24:7455-64. doi: 10.1038/sj.onc.1209085

24. Quispel-Janssen JM, Badhai J, Schunselaar L, Price S, Brammeld J, Iorio $\mathrm{F}$, et al. Comprehensive pharmacogenomic profiling of malignant pleural mesothelioma identifies a subgroup sensitive to FGFR inhibition. Clin Cancer Res. (2018) 24:84-94. doi: 10.1158/1078-0432.CCR17-1172

25. Kolluri KK, Alifrangis C, Kumar N, Ishii Y, Price S, Michaut M, et al. Loss of functional BAP1 augments sensitivity to TRAIL in cancer cells. eLife. (2018) 7:e30224. doi: 10.7554/eLife.30224

26. LaFave LM, Beguelin W, Koche R, Teater M, Spitzer B, Chramiec A, et al. Loss of BAP1 function leads to EZH2-dependent transformation. Nat Med. (2015) 21:1344-9. doi: 10.1038/nm.3947

27. Delage B, Fennell DA, Nicholson L, McNeish I, Lemoine NR, Crook T, et al. Arginine deprivation and argininosuccinate synthetase expression in the treatment of cancer. Int J Cancer. (2010) 126:2762-72. doi: 10.1002/ijc.25202

28. Yap TA, Aerts JG, Popat S, Fennell DA. Novel insights into mesothelioma biology and implications for therapy. Nat Rev Cancer. (2017) 17:47588. doi: $10.1038 /$ nrc. 2017.42

29. Parrotta R, Okonska A, Ronner M, Weder W, Stahel R, Penengo L, et al. A novel BRCA1-associated protein-1 isoform affects response of mesothelioma cells to drugs impairing BRCA1-mediated DNA repair. J Thorac Oncol. (2017) 12:1309-19. doi: 10.1016/j.jtho.2017.03.023

30. Illei PB, Ladanyi M, Rusch VW, Zakowski MF. The use of CDKN2A deletion as a diagnostic marker for malignant mesothelioma in body cavity effusions. Cancer. (2003) 99:51-6. doi: 10.1002/cncr.10923

31. Mavrakis KJ, McDonald ER 3rd, Schlabach MR, Billy E, Hoffman GR, deWeck A, et al. Disordered methionine metabolism in MTAP/CDKN2Adeleted cancers leads to dependence on PRMT5. Science. (2016) 351:120813. doi: $10.1126 /$ science.aad5944

32. Kryukov GV, Wilson FH, Ruth JR, Paulk J, Tsherniak A, Marlow SE, et al. MTAP deletion confers enhanced dependency on the PRMT5 arginine methyltransferase in cancer cells. Science. (2016) 351:12148. doi: $10.1126 /$ science.aad5214

33. Moroishi T, Hansen CG, Guan KL. The emerging roles of YAP and TAZ in cancer. Nat Rev Cancer. (2015) 15:73-9. doi: 10.1038/nrc3876

34. Cheung M, Menges CW, Testa JR. In: Testa JR, editor. Asbestos and Mesothelioma. Heidelberg: Springer Verlag (2017). p. 175-95. doi: 10.1007/978-3-319-53560-9_8

35. Cicala C, Pompetti F, Carbone M. SV40 induces mesothelioma in hamsters. Am J Pathol. (1993) 142:1524-33.

36. Craighead JE, Akley NJ, Gould LB, Libbus GL. Characteristics of tumors and tumor cells cultured from experimental asbestos-induced mesotheliomas in rats. Am J Path. (1987) 24:448-62.

37. Libbus BL, Craighead JE. Chromosomal translocations with specific breakpoints in asbestos-induced rat mesotheliomas. Cancer Res. (1988) 48:6455-61.

38. Marsella JM, Liu BL, Vaslet CA, Kane AB. Susceptibility of $p 53$-deficient mice to induction of mesothelioma by crocidolite asbestos fibers. Environ Health Perspect. (1997) 105:1069-72. doi: 10.2307/3433511

39. Sandhu H, Dehnen W, Roller M, Abel J, Unfried K. mRNA expression patterns in different stages of asbestos-induced carcinogenesis in rats. Carcinogenesis. (2000) 21:1023-9. doi: 10.1093/carcin/21.5.1023

40. Wagner JC, Berry G. Mesotheliomas in rats following inoculation with asbestos. Br J Cancer. (1969) 23:567-81. doi: 10.1038/bjc.1969.70

41. Baris YI, Sahin AA, Ozesmi M, Kerse I, Ozen E, Kolacan B, et al. An outbreak of pleural mesothelioma and chronic fibrosing pleurisy in the village of Karain/Urgup in Anatolia. Thorax. (1978) 33:18192. doi: 10.1136/thx.33.2.181

42. Carbone M, Emri S, Dogan AU, Steele I, Tuncer M, Pass $\mathrm{HI}$, et al. A mesothelioma epidemic in Cappadocia: scientific 
developments and unexpected social outcomes. Nat Rev Cancer. (2007) 7:147-54. doi: $10.1038 / \mathrm{nrc} 2068$

43. Wagner JC, Skidmore JW, Hill RJ, Griffiths DM. Erionite exposure and mesotheliomas in rats. $\mathrm{Br} J$ Cancer. (1985) 51:727-30. doi: 10.1038/bjc.1985.108

44. Davis JM. The long term fibrogenic effects of chrysotile and crocidolite asbestos dust injected into the pleural cavity of experimental animals. BrJ Exp Pathol. (1970) 51:617-27.

45. Reeves AL, Puro HE, Smith RG. Inhalation carcinogenesis from various forms of asbestos. Environ Res. (1974) 8:178202. doi: 10.1016/0013-9351(74)90050-4

46. Suzuki Y, Kohyama N. Malignant mesothelioma induced by asbestos and zeolite in the mouse peritoneal cavity. Environ Res. (1984) 35:27792. doi: 10.1016/0013-9351(84)90136-1

47. Vaslet CA, Messier NJ, Kane AB. Accelerated progression of asbestos-induced mesotheliomas in heterozygous p53(+/-) mice. Toxicol Sci. (2002) 68:3318. doi: $10.1093 /$ toxsci/68.2.331

48. Fleury-Feith J, Lecomte C, Renier A, Matrat M, Kheuang L, Abramowski V, et al. Hemizygosity of $N f 2$ is associated with increased susceptibility to asbestos-induced peritoneal tumours. Oncogene. (2003) 22:3799-805. doi: $10.1038 /$ sj.onc. 1206593

49. Robinson C, van Bruggen I, Segal A, Dunham M, Sherwood A, Koentgen F, et al. A novel SV40 TAg transgenic model of asbestos-induced mesothelioma: malignant transformation is dose dependent. Cancer Res. (2006) 66:1078694. doi: 10.1158/0008-5472.CAN-05-4668

50. Altomare DA, Menges CW, Pei J, Zhang L, Skele-Stump KL, Carbone $\mathrm{M}$, et al. Activated TNF-alpha/NF-kappaB signaling via down-regulation of Fas-associated factor 1 in asbestos-induced mesotheliomas from Arf knockout mice. Proc Natl Acad Sci USA. (2009) 106:3430-5. doi: 10.1073/pnas.0808816106

51. Altomare DA, Menges CW, Xu J, Pei J, Zhang L, Tadevosyan A, et al. Losses of both products of the Cdkn2a/Arf locus contribute to asbestos-induced mesothelioma development and cooperate to accelerate tumorigenesis. PLoS ONE. (2011) 6:e18828. doi: 10.1371/journal.pone.0018828

52. Chow MT, Tschopp J, Möller A, Smyth MJ. NLRP3 promotes inflammationinduced skin cancer but is dispensable for asbestos-induced mesothelioma. Immunol Cell Biol. (2012) 90:983-6. doi: 10.1038/icb.2012.46

53. Xu J, Kadariya Y, Cheung M, Pei J, Talarchek J, Sementino E, et al. Germline mutation of Bap1 accelerates development of asbestos-induced malignant mesothelioma. Cancer Res. (2014) 74:4388-97. doi: 10.1158/0008-5472.CAN-14-1328

54. Kadariya Y, Cheung M, Xu J, Pei J, Sementino E, Menges CW, et al. Bap1 is a bona fide tumor suppressor: genetic evidence from mouse models carrying heterozygous germline Bap1 mutations. Cancer Res. (2016) 76:283644. doi: 10.1158/0008-5472.CAN-15-3371

55. Kadariya Y, Menges CW, Talarchek J, Cai KQ, Klein-Szanto AJ, Pietrofesa RA, et al. Inflammation-related IL1B/IL1R signaling promotes the development of asbestos-induced malignant mesothelioma. Cancer Prev Res. (2016) 9:40614. doi: 10.1158/1940-6207.CAPR-15-0347

56. Menges CW, Kadariya Y, Altomare D, Talarchek J, Neumann-Domer E, $\mathrm{Wu} \mathrm{Y}$, et al. Tumor suppressor alterations cooperate to drive aggressive mesotheliomas with enriched cancer stem cells via a p53-miR-34a-c-Met axis. Cancer Res. (2014) 74:1261-71. doi: 10.1158/0008-5472.CAN-13-2062

57. Donehower LA, Harvey M, Vogel H, McArthur MJ, Montgomery CA Jr, Park $\mathrm{SH}$, et al. Effects of genetic background on tumorigenesis in p53-deficient mice. Mol Carcinog. (1995) 14:16-22. doi: 10.1002/mc.2940140105

58. Walpole S, Pritchard AL, Cebulla CM, Pilarski R, Stautberg M, Davidorf $\mathrm{FH}$, et al. Comprehensive study of the clinical phenotype of germline BAP1 variant-carrying families worldwide. J Natl Cancer Inst. (2018) 110:132841. doi: 10.1093/jnci/djy171

59. Napolitano A, Pellegrini L, Dey A, Larson D, Tanji M, Flores EG, et al. Minimal asbestos exposure in germline BAP1 heterozygous mice is associated with deregulated inflammatory response and increased risk of mesothelioma. Oncogene. (2016) 35:1996-2002. doi: 10.1038/onc.2015.243

60. Robinson C, Walsh A, Larma I, O'Halloran S, Nowak AK, Lake RA. MexTAg mice exposed to asbestos develop cancer that faithfully replicates key features of the pathogenesis of human mesothelioma. Eur J Cancer. (2011) 47:15161. doi: 10.1016/j.ejca.2010.08.015
61. Lopez-Rios F, Illei PB, Rusch V, Ladanyi M. Evidence against a role for SV40 infection in human mesotheliomas and high risk of false-positive PCR results owing to presence of SV40 sequences in common laboratory plasmids. Lancet. (2004) 364:1157-66. doi: 10.1016/S0140-6736(04)17102-X

62. Hübner R, Van Marck E. Reappraisal of the strong association between simian virus 40 and human malignant mesothelioma of the pleura (Belgium). Cancer Causes Control. (2002) 13:121-9. doi: 10.1023/A:1014321729038

63. Robinson C, Dick IM, Wise MJ, Holloway A, Diyagama D, Robinson BW, et al. Consistent gene expression profiles in MexTAg transgenic mouse and wild type mouse asbestos-induced mesothelioma. BMC Cancer. (2015) 15:983. doi: 10.1186/s12885-015-1953-y

64. Jongsma J, van Montfort E, Vooijs M, Zevenhoven J, Krimpenfort P, van der Valk M, et al. A conditional mouse model for malignant mesothelioma. Cancer Cell. (2008) 12:261-71. doi: 10.1016/j.ccr.2008.01.030

65. Akagi K, Sandig V, Vooijs M, Van der Valk M, Giovannini M, Strauss M, et al. Cre-mediated somatic site-specific recombination in mice. Nucleic Acids Res. (1997) 25:1766-73. doi: 10.1093/nar/25.9.1766

66. Ohar JA, Cheung M, Talarchek J, Howard SE, Howard TD, Hesdorffer M, et al. Germline BAP1 mutational landscape of asbestos-exposed malignant mesothelioma patients with family history of cancer. Cancer Res. (2016) 76:206-15. doi: 10.1158/0008-5472.CAN-15-0295

67. Harbour JW, Onken MD, Roberson ED, Duan S, Cao L, Worley LA, et al. Frequent mutation of BAP1 in metastasizing uveal melanomas. Science. (2010) 330:1410-3. doi: 10.1126/science.1194472

68. Pena-Llopis S, Vega-Rubin-de-Celis S, Liao A, Leng N, Pavia-Jimenez A, Wang S, et al. BAP1 loss defines a new class of renal cell carcinoma. Nat Genet. (2012) 44:751-9. doi: 10.1038/ng.2323

69. Kukuyan AM, Sementino E, Kadariya Y, Menges CW, Cheung $\mathrm{M}$, Tan $\mathrm{Y}$, et al. Inactivation of Bap1 cooperates with losses of Nf2 and Cdkn2a to drive the development of pleural malignant mesothelioma in conditional mouse models. Cancer Res. (2019) 79:4113-23. doi: 10.1158/0008-5472.CAN-18-4093

70. Sementino E, Menges CW, Kadariya Y, Peri S, Xu J, Liu Z, et al. Inactivation of $T p 53$ and Pten drives rapid development of pleural and peritoneal malignant mesotheliomas. J Cell Physiol. (2018) 233:8952-61. doi: 10.1002/jcp.26830

71. Zhao CY, Grinkevich VV, Nikulenkov F, Bao W, Selivanova G. Rescue of the apoptotic-inducing function of mutant $\mathrm{p} 53$ by small molecule RITA. Cell Cycle. (2010) 9:1847-55. doi: 10.4161/cc.9.9.11545

72. Wu L, Allo G, John T, Li M, Tagawa T, Opitz I, et al. Patient-derived xenograft establishment from human malignant pleural mesothelioma. Clin Cancer Res. (2017) 23:1060-7. doi: 10.1158/1078-0432.CCR16-0844

73. Nabavi N, Wei J, Lin D, Collins CC, Gout PW, Wang Y. Pre-clinical models for malignant mesothelioma research: from chemical-induced to patient-derived cancer xenografts. Front Genet. (2018) 9:232. doi: 10.3389/fgene.2018.00232

74. Herndler-Brandstetter D, Shan L, Yao Y, Stecher C, Plajer V, Lietzenmayer $\mathrm{M}$, et al. Humanized mouse model supports development, function, and tissue residency of human natural killer cells. Proc Natl Acad Sci USA. (2017) 114:E9626-34. doi: 10.1073/pnas.1705301114

75. Servais EL, Colovos C, Kachala SS, Adusumilli PS. Pre-clinical mouse models of primary and metastatic pleural cancers of the lung and breast and the use of bioluminescent imaging to monitor pleural tumor burden. Curr Protoc Pharmacol. (2011) 54:14.21.1-18. doi: 10.1002/0471141755.ph1421s54

Conflict of Interest: JT has a patent on BAP1 mutation testing and has provided legal consultation regarding the role of germline mutations of BAP1 in mesothelioma.

The remaining author declares that the research was conducted in the absence of any commercial or financial relationships that could be construed as a potential conflict of interest.

Copyright (c) 2020 Testa and Berns. This is an open-access article distributed under the terms of the Creative Commons Attribution License (CC BY). The use, distribution or reproduction in other forums is permitted, provided the original author(s) and the copyright owner(s) are credited and that the original publication in this journal is cited, in accordance with accepted academic practice. No use, distribution or reproduction is permitted which does not comply with these terms. 